\title{
QUALITY ASSESSMENT FOR ONLINE IRIS IMAGES
}

\author{
Sisanda Makinana, Tendani Malumedzha, and Fulufhelo V Nelwamondo \\ Modelling and Digital Science, CSIR, Pretoria, South Africa \\ smakinana@csir.co.za
}

\begin{abstract}
Iris recognition systems have attracted much attention for their uniqueness, stability and reliability. However, performance of this system depends on quality of iris image. Therefore there is a need to select good quality images before features can be extracted. In this paper, iris quality is done by assessing the effect of standard deviation, contrast, area ratio, occlusion, blur, dilation and sharpness on iris images. A fusion method based on principal component analysis (PCA) is proposed to determine the quality score. CASIA, IID and UBIRIS databases are used to test the proposed algorithm. SVM was used to evaluate the performance of the proposed quality algorithm. . The experimental results demonstrated that the proposed algorithm yields an efficiency of over $84 \%$ and $90 \%$ Correct Rate and Area under the Curve respectively. The use of character component to assess quality has been found to be sufficient for quality detection.
\end{abstract}

\section{KEYWORDS}

Image quality, Iris recognition, Principal Component Analysis, Support Vector Machine.

\section{INTRODUCTION}

Iris recognition is an automated method of biometric identification that analyses patterns of the iris to identify an individual [1]. It is said to have high reliability in identification because each individual has unique iris patterns [2], [3]. However, due to the limited effectiveness of imaging, it is important that image of high-quality images are selected in order to ensure reliable human identification. Some advanced pre-processing algorithms can process poor quality images and produce adequate results, however they are computationally expensive and add extra burden on the recognition system time. Therefore quality determination is necessary in order to determine which algorithm to use for pre-processing. For example, if it's known that the acquired image does not meet the desired quality it can be subjected to stricter pre-processing algorithms selectively. Various quality assessment methods have been developed to ensure quality of the sample acquisition process for online systems [4]. These approaches are good for quick elimination of poor quality images and even images from which an accurate segmentation may be produced are eliminated. A more discriminative approach to quality, images can be assigned quality levels, which will provide an indication as to whether further processing can enhance them.

Generally, an iris sample is of good quality if it provides enough features for reliable identification of an individual [5]. Therefore, there is need for a standard sample quality that 
stipulates the accepted quality for iris images. In this regard, ISO/IEC have developed three quality components which together defines biometric sample quality, these are; character, fidelity and utility [6]. In this paper, the focus is on character component of a biometric sample quality due to the fact that available algorithms utilises and focuses on fidelity and utility components [4], [7], [8]. This paper proposes an algorithm that assesses the quality of an iris image based on online biometric systems. Firstly, image quality parameters are estimated, i.e. contrast, sharpness, blur, dilation, area ratio, standard deviation and occlusion. Thereafter, a fusion technique based on principal component analysis is used to weight each quality parameter and obtain a quality score for each image. The remainder of this paper is organised as follows. Section II describes the overview of the proposed method. Section III provides the estimations of individual quality parameters and discusses the implementation of the proposed fusion method. Last sections provide experimental results and a conclusion.

\section{ESTIMATION OF QUALITY PARAMETERS}

Implementation of assessment algorithm is carried out in two steps: namely, iris segmentation and estimation and fusion of quality parameters. The subsections below details how this is done.

\subsection{Iris Segmentation}

To locate the iris from the acquired eye image, parameters (radius $\mathrm{r}$, and the coordinates of the centre of the circle, $\mathrm{x}_{0}$ and $\mathrm{y}_{0}$ ) of detecting the centre of the iris and pupil were determined by use of integrodifferential operator discussed in [2]. This operator locates and segments the pupil and iris regions with varying coordinates. Equation 1 describes the integrodifferential operator:

$$
\max \left(r, x_{0}, y_{0}\right)\left|G_{\sigma}(r) * \frac{\partial}{\partial r} \oint_{r, x_{0}, y_{0}} \frac{I(x, y)}{2 \pi r} d s\right|
$$

Where $G_{\sigma}(r)$ is the Gaussian kernel, $I(x, y)$ is the eye image, $\left(x_{0}, y_{0}\right), r$, are the centre coordinates and radius of the circle respectively.

\subsection{Estimation of quality parameters}

The following are the quality parameters that are estimated for the proposed algorithm:

\subsubsection{Occlusion}

The occlusion measure $\left(\mathrm{M}_{\mathrm{O}}\right)$ is the amount of iris region that is invalid due to obstruction by eyelids and eyelashes. Eyelid and eyelashes occlusion problem is a primary cause of bad quality in iris image [9]. Compared with the edge of iris texture, the edge of iris-lid and iris-lash is much sharper and usually considered to contain high pixel values [9]. To estimate the amount of occlusion at each level an occlusion is measured by calculating the ratio of total gray value of the image [9]. It is defined as:

$$
\begin{aligned}
& T_{G}=A_{I} \times \sum_{i=1}^{m} \sum_{j=1}^{n} I \\
& G_{R}=\frac{1}{T_{G}}
\end{aligned}
$$




$$
M_{O}=\operatorname{mean}\left(G_{R}\right)
$$

Where in (2) $A_{I}$ and $I$ is the area of the iris and the iris intensity, $m$ and $n$ represents the size of the image and TG is the total gray value. In (3) $T_{G}$ is the ratio of the total gray value. The higher the metric value the greater is the chance for occlusion by iris lid.

\subsubsection{Blur}

Blur may result from many sources, but in general it occurs when the focal point is outside the depth of field of the captured object [9]. The further the object is from the focal point of the capturing device, the higher degree of blur in the output image [10]. The blur estimation is based on Crete et al [11] approach. The blur estimation procedure is illustrated in Fig. 1.

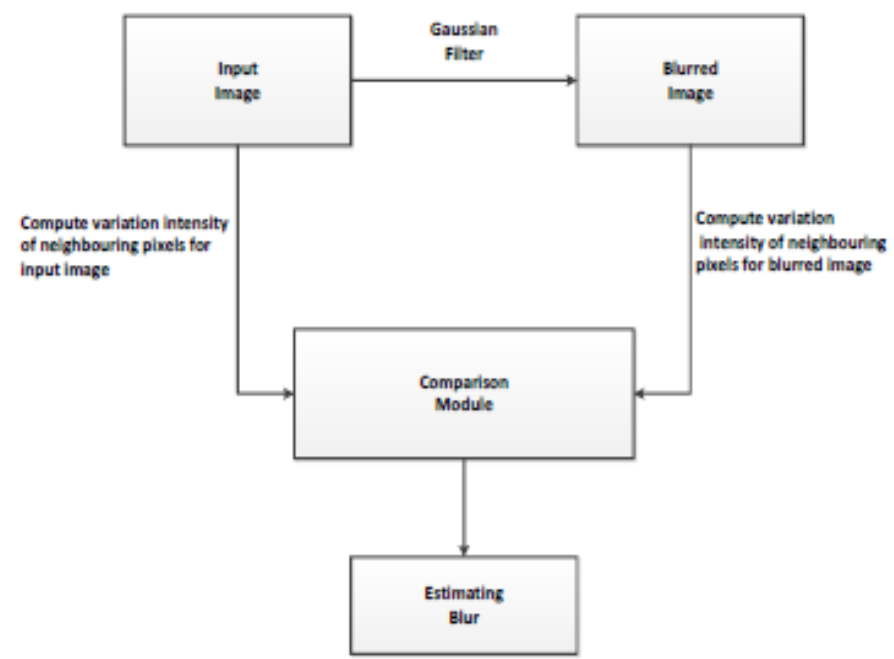

Figure 1 The framework of the blur estimation [9]

\subsubsection{Area Ratio}

The representation of pattern recognition should be invariant to change in the size, position and orientation of the iris image. If the subject is too close to the capturing device that may cause the captured image to be blurred. Thus, it's of utmost importance to assess the iris area ratio, which is the ratio of the iris over the image frame [9]. It is assumed that the iris is circular, therefore the area of the iris is equivalent to the area of the circle given in (4) which is then defined as [9]

$$
A_{I}=\pi r^{2}
$$

The area of the image frame is given as:

$$
A_{E}=H * W
$$

Where $\mathrm{H}$ is the height and $\mathrm{W}$ is width of the image. Therefore the area ratio is derived as:

$$
M_{A}=\frac{A_{I}}{A_{E}}
$$




\subsubsection{Contrast}

The term contrast refers to the representation of colour and difference in luminance that makes a feature noticeable within an image [12]. However human vision is more sensitive to difference in colour representation than difference in luminance. According to human visual, contrast is the difference in colour and brightness of various objects within the same field of view. Contrast determines the clearness of the features within an image. High contrast means the more clearly the iris features and making easier for feature extraction. Assessing contrast is important to ensure sufficient and clear features are extracted. In measuring contrast a window of $8 \times 8$ pixels is defined, of which the Fast Fourier Transform (FFT) of the 2-Dimensional image is computed for each sub-window. FFT transforms the signal from time domain into a frequency domain and is defined as [13]:

$$
H(u, v)=\sum_{x=0}^{M-1} \sum_{y=0}^{m-1} \exp \left[-2 \pi i y \frac{v}{M}\right] \exp \left[-2 \pi i x \frac{u}{M}\right] h(x, y)
$$

Where $u, v=-\frac{M}{2} \ldots \ldots \frac{M}{2}$. The squared magnitude of the Fourier series coefficients which indicates power at corresponding frequencies is computed by Parseval's Theorem [14]:

$$
\mathrm{P}(\mathrm{u}, \mathrm{v})=|H(u, v)|^{2}=(\operatorname{Re}(u, v)+\operatorname{Im}(u, v))^{2}
$$

The fundamental frequency (dcpower), the total power (totpower) and the non-zero power (acpower) of the spectrum is computed which are [14]:

$$
\begin{gathered}
\text { dcpower }=P(0,0)=|H(0,0)|^{2} \\
\text { totpower }=\sum_{u=-M}^{M} \sum_{v=-M}^{M} P(u, v) \\
\text { acpower }=\text { totpower }- \text { dcpower }
\end{gathered}
$$

The contrast is computed as follows [14]:

$$
M_{C}=\sqrt{\frac{\text { acpower }}{\text { dcpower }}}
$$

\subsubsection{Standard Deviation}

Standard deviation method uses the standard deviation of gray- level distribution in a local area region of an iris image. The iris image is divided into $\mathrm{N} \mathrm{X} \mathrm{N}$ regions. The local standard deviation of each region is computed and added together to obtain a single standard deviation. Then, the mean of the summed standard deviation is quality score of the entire image.

$$
S_{k}=\sqrt{\frac{1}{N^{2}} \sum_{x=1}^{N} \sum_{y=1}^{N}\left(I_{x y}-I_{k}\right)^{2}}
$$




$$
M_{S T D}=\frac{1}{N} \sum_{k=1}^{N} S_{k}
$$

In (13), $I_{x, y}$ is the gray level of pixel $(x, y)$ and $I_{k}$ is average gray level of the $k^{\text {th }}$ region.

\subsubsection{Sharpness}

A sharp image is the one that contains fine details that determine the amount of detail an image can produce and has edges and objects appearing to be of high contrast. Images are usually affected by distortions during acquisition and processing, which may result in loss of visual quality. Therefore, image sharpness assessment is useful in such application. Sharpness generally attenuates high frequencies. Due to that factor, sharpness can be assessed by measuring high frequency of the image. Daugman [4] proposed an 8 X 8 convolution kernel to assess sharpness. Sharpness is estimated based on the gradient of the image to determine whether the image is in focus or not, because the gradient of an image is the directional change in the intensity of an image. The gradient of the image is given by:

$$
\nabla G=\left(\frac{\partial G^{\wedge}}{\partial x} x\right)+\left(\frac{\partial G^{\wedge}}{\partial y} y^{\wedge}\right)
$$

Where $\frac{\partial G}{\partial x}$ is the gradient in the $\mathrm{x}$ direction and $\frac{\partial G}{\partial y}$ is the gradient in the y direction. From (15) the sharpness of the image may be calculated. The sharpness is calculated by dividing the sum of gradient amplitude by the number of elements of the gradient. The gradient amplitude is given by:

$$
M_{S}=S \sqrt{G_{x}^{2}+G_{y}^{2}}
$$

Where $\mathrm{S}$ is the gradient amplitude, $G_{x}$ and $G_{y}$ in (16) are the horizontal and vertical change in intensity.

\subsubsection{Dilation:}

The variation in pupil dilation between the enrolment image and the image to be recognised or verified may affect the accuracy of iris recognition system [9]. The degree of dilation was measured for each iris image. The segmentation results provided the radius of the pupil and of the iris. To measure dilation, a ratio of radius of pupil and radius of iris was calculated. Since the pupil radius is always less than the radius of iris, the dilation will fall between 0 and 1 [9]. The dilation measure $M_{D}$ is calculated by:

$$
M_{D}=\frac{P_{R}}{I_{R}}
$$

Where $P_{R}$ is the pupil radius and $I_{R}$ is the iris radius.

\section{FUSION TECHNIQUE}

A unique quality score is of value to the prediction step of iris recognition system. To obtain this quality score, a fusion technique based on Principal Component Analysis (PCA) is proposed. PCA is a widely used tool which is proficient in reducing dimensions and determining factor 
relationships amongst datasets just like Factor Analysis (FA) [15]. However, FA evaluates the linear relationship between the number of variables of interest $Y_{1}, Y_{2} \ldots . . Y_{j}$; and a smaller number of unobserved factors $F_{1}, F_{2}, \ldots \ldots . . F_{k}$, whereas, PCA is a technique that determines the factor loadings of the dataset by calculating the eigenvectors and eigenvalues of the covariance matrix [16]. In this research PCA has been used over FA since the interest in determining the factor loading of the dataset. Factor loadings are the weights of each variable and correlations between each factor [17]. The PCA is calculated by defining the eigenvectors and eigenvalues of the covariance matrix. The covariance matrix measures the variation of the dimensions from the mean with respect to each other. Prior to applying the PCA, quality parameters need to be normalised. The quality parameters are standardized using the $Z_{S}$ before obtaining the first PCA, which is:

$$
Z_{S}=\frac{x-\mu}{\sigma}
$$

Where $\mu$ the mean and $\sigma$ is the standard deviation of the estimated measures of the entire database. Suppose $n$ independent observation are given on $X_{1}, X_{2} \ldots . X_{k}$, where the covariance $X_{i}$ and $X_{j}$ is

$$
\operatorname{Cov}\left(X_{i}, X_{j}\right)=\sum i, j
$$

For $i, j=1,2 \ldots ., k$ in (19). Then the eigenvalues and eigenvectors of the covariance matrix are calculated. $\mathrm{W}$ is defined to be the first principal component. It is the linear combination of the X"s with the largest variance:

$$
W=a_{1}^{T} X_{i}
$$

Where $i=1,2, \ldots \ldots ., k$ and $a$ is the eigenvector of the covariance. The quality score is obtained by multiplying normalised measures of parameters with weights for each quality parameter of the image. The fusion quality index is given as:

$$
Q_{S}=\sum_{i=1}^{N} Q_{p} W_{p}
$$

Where $Q_{S}$ the quality score, $Q_{P}$ is the estimated quality parameter and $W_{p}$ is the amount of influence each parameter has on the quality score. The scores represent the global quality score of the iris segmented images.

\section{QUALITY SCORE NORMALIZATION}

Prior to fusion of the parameters to form a quality score, some parameters need to be normalized between $[0,1]$. Sharpness, Area Ratio, dilation and blur are already in the desired score range. Occlusion is normalized based on the max normalization. The fused score also needed to be normalized between $[0,1]$ with 0 implying bad quality and 1 good quality. The normalization of the quality score is based on the modified form of min-max normalization:

$$
Q_{S}=\frac{Q_{\text {old }}-Q_{\min }}{Q_{\max }-Q_{\min }}
$$


With $Q_{\text {old }}$ represents the raw quality score.

\section{DATASET USED FOR ANALYSIS}

In this paper, the CASIA and UBIRIS databases which are available free online and Internal Iris Database (IID) database were used, to estimate the quality parameters and their scores. For CASIA a subset of images called 'interval' was used. It contained 525 images which were captured at a resolution of $320 \times 280$ pixels. UBIRIS consists of 802 images captured at a resolution of $200 \times 150$ pixels. IID consists of 116 images captured at a resolution of $640 \times 480$ pixels.

Table I DATABASE DESCRIPTION

\begin{tabular}{|l|l|l|l|}
\hline Database & Image No. & Bad Quality & Good Quality \\
\hline UBIRIS & 802 & 685 & 117 \\
\hline CASIA & 525 & 69 & 456 \\
\hline IID & 116 & 35 & 81 \\
\hline
\end{tabular}

\section{IMAGE DESCRIPTION}

For UBIRIS database images were captured on two different sessions. For the first session noise factors like reflection, luminosity and contrast were minimized by capturing images inside a dark room. In the second session capturing location was changed to introduce noisy images. This introduced diverse images with respect to reflection, contrast, focus and luminosity problems [18].

For CASIA database images were captured by a closed up iris camera with circular NIR LightEmitting Diode (LED) array which had suitable luminous flux for iris imaging. The camera captures very clear iris images [19].

The IID iris database which was also used for testing the algorithm is a new database of which its images were collected in the Council of Scientific and Industrial Research (CSIR) campus in Pretoria. A Vista EY2 iris camera was used to collect these images.

\section{EXPERIMENTAL RESULTS}

Analysis was performed on three databases, namely, CASIA Interval, UBIRIS and IID. The sample iris images in Fig. 2 are from UBIRIS, IID and CASIA databases. Based on human visual assessment sample (a) represents good quality from UBIRIS database and (c) represent good quality from IID database. Sample (e) and (f) represent good and bad quality respectively, from CASIA Interval database. Image (b) and (f) represent degraded image quality that is affected by occlusion, blur and dilation. Sample image (b) is also affected by area ratio quality parameter. Table II illustrates the estimated quality parameters of the images in Fig. 2. The quality scores are normalized to the values between zero and one, with one implying good quality and 0 bad quality. The overall quality distribution for CASIA, UBIRIS and IID databases are illustrated in Fig. 3 respectively. CASIA has the highest quality distribution, followed by IID and the UBIRIS. IID suffers from quality degrading with respect to sharpness, dilation and blur, which is visually evident. These parameters have high weight on the quality score of IID which results in low quality. The reason for this problem is the mere fact that the iris capturing session for this database was done in an environment with light which caused reflections, resulting in the images 
being less clear. Moreover, individuals were required to focus their eyes to a mirror for a certain period which caused their pupil to dilate. Also, the camera captured iris images automatically and required individuals to be still which caused some discomfort and as the individual became tired and moved, which resulted in the camera capturing blurred images. For the UBIRIS database was captured in an environment that introduced noisy images affected by diverse problems with respect to reflection, contrast, focus and luminosity. The results of individual parameters also indicate that this database is affected by sharpness, dilation, area ratio and blur which is caused by the environment condition. That is why there are more low quality scores for this database. When grading these data sets in terms of quality scores obtained on the plots, CASIA scores the highest, followed by IID and then UBIRIS.

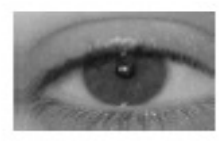

(a)

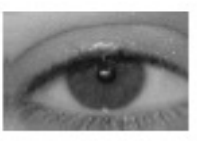

(b)

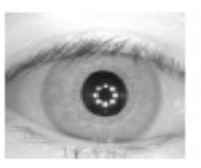

(e)

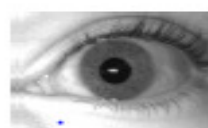

(c)

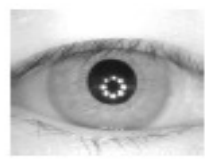

(f)

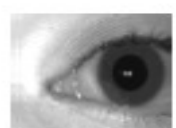

(d)

Figure 2 Sample eye images from UBIRIS, IID and CASIA Interval database. (a) - (b) UBIRIS. (c) - (d) IID. (e) - (f) CASIA.

Table II ESTIMATED QUALITY PARAMETERS OF IMAGES IN FIG. 2

\begin{tabular}{|l|l|l|l|l|l|l|l|}
\hline $\mathbf{M}_{\mathbf{C}}$ & $\mathbf{M}_{\mathbf{S T D}}$ & $\mathbf{M}_{\mathbf{O}}$ & $\mathbf{M}_{\mathbf{S}}$ & $\mathbf{M}_{\mathbf{A}}$ & $\mathbf{M}_{\mathbf{D}}$ & $\mathbf{M}_{\mathbf{B}}$ & Score \\
\hline 0.2755 & 0.9897 & 0.4137 & 0.0271 & 0.0729 & 0.5182 & 0.3242 & 0.8089 \\
\hline 0.2841 & 0.7013 & 0.0316 & 0.0502 & 0.0636 & 0.4352 & 0.4134 & 0.0209 \\
\hline 0.3375 & 0.8273 & 0.0707 & 0.0052 & 0.6773 & 0.3714 & 0.3103 & 0.8930 \\
\hline 0.3372 & 0.9810 & 0.3574 & 0.0299 & 0.5958 & 0.4222 & 0.285 & 0.3471 \\
\hline 0.2535 & 0.7456 & 0.2927 & 0.0252 & 0.6689 & 0.3818 & 0.3212 & 0.8339 \\
\hline 0.2718 & 0.8113 & 0.7552 & 0.0711 & 0.0229 & 0.4286 & 0.2924 & 0.5768 \\
\hline
\end{tabular}
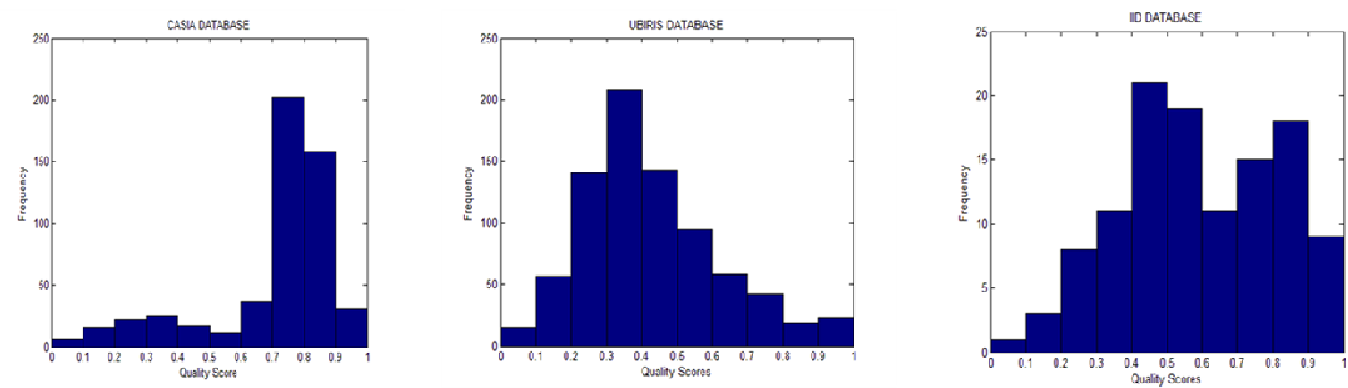

Figure 3 Overall Quality Distribution of CASIA, UBIRIS and IID Databases

\section{Classification Performance}

The performance of the biometric system is typically characterized by error rate. To evaluate the separation performance of the good and bad images of the proposed quality metric, a k-fold cross- 
validation technique is employed in a Support Vector Machine (SVM) classifier. SVM is a classifier that performs classification by constructing a hyperplane in a multidimensional space and separating the data points into different classes [20]. K-fold cross-validation is a rotational estimation of which dataset is randomly grouped into k mutually exclusive folds of approximately equal size [21]. Data is divided into k groups and each group is rotated between being a training group and a testing group. In this research $\mathrm{k}=10$ and then the correct rate of the classifier is averaged out. The performance of the assessment is illustrated in Table III. From these results it is clear that the proposed assessment algorithm is significant as the correct rate is above $80 \%$ on both classifiers.

Table III SUMMARY OF PERFORMANCE STATISTICS USING SVM

\begin{tabular}{|l|l|l|}
\hline Database & Correct Rate & Error Rate \\
\hline CASIA & 99.05 & 0.95 \\
\hline UBIRIS & 98.75 & 1.25 \\
\hline IID & 84.48 & 15.52 \\
\hline
\end{tabular}

\section{CONFUSION MATRIX}

In CASIA database out of 525 images only 5 were classified wrongly giving an overall average total of $99.05 \%$ accuracy in performance of the classifier. For UBIRIS database none of the images were classified as bad quality while they were actually good quality and 10 images were classified as good quality while they were bad, giving an overall average total of $98.75 \%$ in classifier accuracy. Last, for IID database out of 116 images only 18 images were classified wrongly giving an overall average total of $84.48 \%$ in accuracy. Table IV, V and VI illustrates these results.

In this research, there is no ground truth for all databases used, so human inspection was used to classify the images. However, humans have limited resolution, so they cannot detect quality of the images pixel by pixel. Moreover, humans perceive quality with limited factors such as clearness of features, blurriness and brightness of the image. On the other hand, the proposed algorithm determines quality based on standard deviation, contrast, dilation, blur, sharpness, area ratio and occlusion by calculating each factor pixel by pixel. Therefore, the proposed algorithm can calculate quality better than the human eye hence the misclassification.

Table IV CONFUSION MATRIX FOR CASIA

\begin{tabular}{|c|c|c|c|}
\cline { 2 - 4 } \multicolumn{1}{c|}{} & \multicolumn{3}{|c|}{ Actual class } \\
\cline { 2 - 4 } \multicolumn{1}{c|}{} & & TP & TN \\
\hline \multirow{3}{*}{ Predicted class } & TP & 69 & 0 \\
\cline { 2 - 4 } & TN & 5 & 451 \\
\hline
\end{tabular}

Table V CONFUSION MATRIX FOR UBIRIS

\begin{tabular}{|c|c|c|c|}
\cline { 2 - 4 } \multicolumn{1}{c|}{} & \multicolumn{3}{|c|}{ Actual class } \\
\cline { 2 - 4 } \multicolumn{1}{c|}{} & & TP & TN \\
\hline \multirow{3}{*}{ Predicted class } & TP & 675 & 10 \\
\cline { 2 - 4 } & TN & 0 & 116 \\
\hline
\end{tabular}


Table VI CONFUSION MATRIX FOR IID

\begin{tabular}{|c|c|c|c|}
\cline { 2 - 4 } \multicolumn{1}{c|}{} & \multicolumn{3}{c|}{ Actual class } \\
\cline { 2 - 4 } \multicolumn{1}{c|}{} & & TP & TN \\
\hline \multirow{2}{*}{ Predicted class } & TP & 35 & 4 \\
\cline { 2 - 4 } & TN & 14 & 63 \\
\hline
\end{tabular}

\section{Prediction Performance of Proposed Assessment METHOD}

Fig. 4, 5 and 6 illustrate the prediction performance of the proposed quality assessment algorithm using the CASIA, UBIRIS and IID databases. The false positive rate of all three databases is low, implying that fewer images were misclassified than correctly classified. Table VII contains the statistics of the ROC curve analysis. The area under the curve for all databases range between 92 $\%$ to $97 \%$, which indicates a good performance of the classifier. Moreover, the $95 \%$ confidence interval (CI) for all databases are fairly high, with UBIRIS having the lowest lower bound of 0.88469 , which implies the performance of the classifier is good. This concludes that the proposed algorithm is capable of distinguishing a good sample quality from a bad one. It can also be observed that the proposed quality assessment method can predict the quality of the image for all three databases as the AUC of the above ROC curves is above $90 \%$. These results imply that the proposed fused quality measure is suitable to be used as the informal measure for ensuring images of sufficient quality are used for feature extraction.

\section{Table VII STATISTICS OF ROC CURVE ANALYSIS}

\begin{tabular}{|l|l|l|l|}
\hline Database & S. E. & AUC & C.I. \\
\hline CASIA & 0.00979 & 0.92505 & 0.925050 .96342 \\
\hline UBIRIS & 0.01127 & 0.96707 & 0.944990 .98915 \\
\hline IID & 0.02299 & 0.92975 & 0.884690 .97480 \\
\hline
\end{tabular}

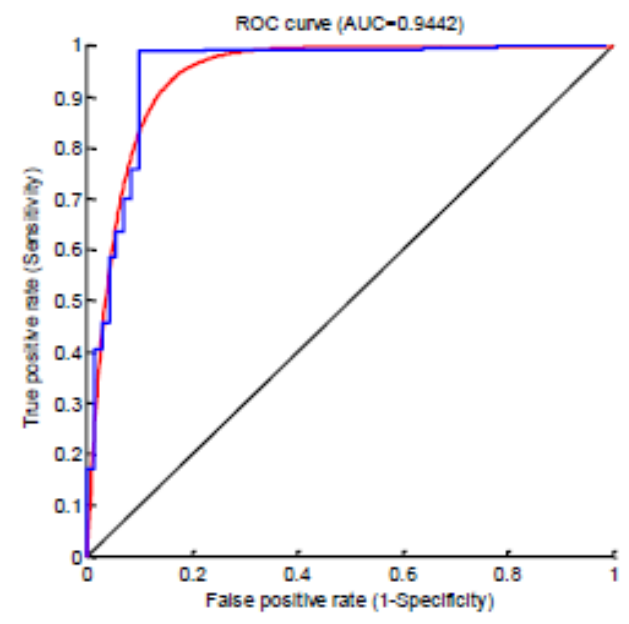

Figure 4 Verification performance of CASIA Database 


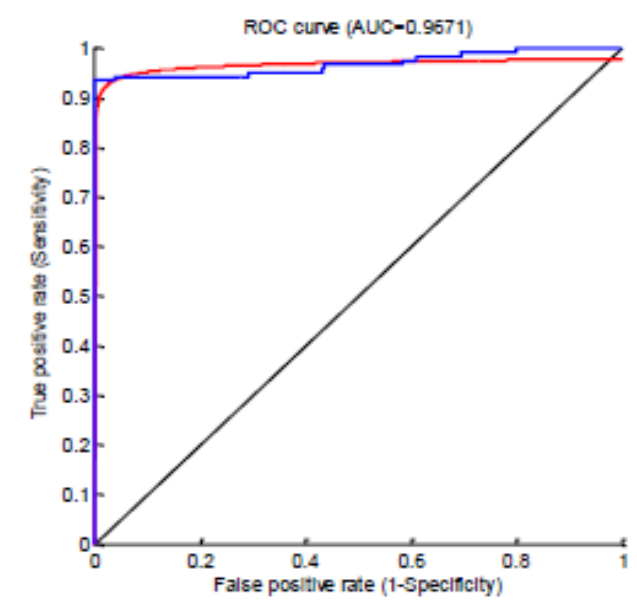

Figure 5 Verification performance of UBIRIS Database

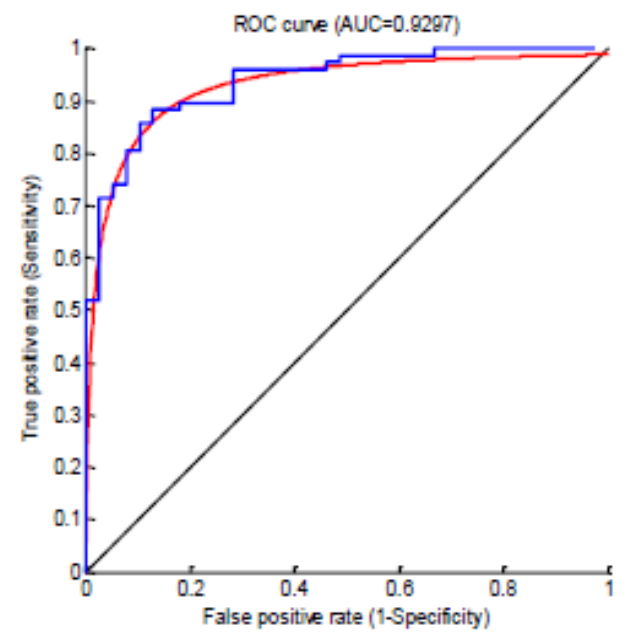

Figure 6 Verification performance of IID Database

\section{CONCLUSION}

In order to guide the selection of image of good quality, a quality model that evaluates the results of segmented iris image based on richness of the texture, shape and amount of information in the iris image has been developed. We extend iris quality assessment research by analysing the effect of various quality parameters such as standard deviation, contrast, area ratio, occlusion, blur, dilation and sharpness of an iris image. A fusion approach is presented for fusing all quality measures to a quality score. This is necessary because in order to successfully identify an individual on iris recognition systems an iris image must have sufficient features for extraction. The aim of this paper is to present a method that could be used for selection of high quality images, which may improve iris recognition performance. In analysing results the proposed assessment method proved to be capable of quality characterisation as it yields above $84 \%$ in CR. The major benefit of this paper is that assessment is done before feature extraction, so only high quality images will be processed therefore saving time and resources. 


\section{ACKNOWLEDGEMENTS}

The authors wish to thank CSIR Modelling and Digital Science for sponsoring this research, without your support I would not be able to accomplish my studies. Also, I want to acknowledge Tendani Malumedzha and Prof Fulufhelo Nelwamondo for their patience, wisdom and guidance. Thank you.

\section{REFERENCES}

[1] Gulmire, K. and Ganorkar, S., (2012), "Iris recognition using Gabor wavelet." International Journal of Engineering, Vol. 1, No. 5.

[2] Masek, L., "Recognition of human iris patterns for biometric identification." $\mathrm{PhD}$ thesis.

[3] Ma, L., Tan, T., Wang, Y. and Zhang, D., (2003) "Personal identification based on iris texture analysis." Pattern Analysis and Machine Intelligence, IEEE Transactions on, Vol. 25, No. 12, pp $1519-1533$.

[4] Daugman, J., (2004), "How iris recognition works." Circuits and Systems for Video Technology, IEEE Transactions on, Vol. 14, No. 1, pp 21-30.

[5] Belcher, C., and Du, Y. (2008), "A selective feature information approach for iris image-quality measure". Information Forensics and Security, IEEE Transactions on, pp572-577.

[6] Tabassi, E., (2009), "Biometric Quality Standards", NIST, Biometric Consortium.,

[7] Fatukasi, O., Kittler, J., and Poh, N., (2007), "Quality controlled multi-modal fusion of biometric experts.", In Progress in Pattern Recognition, Image Analysis and Applications, pp 881-890. Springer.

[8] Kalka,N. D., Dorairaj, V., Shah,Y. N., Schmid, N. A. and Cukic B.,( 2002), “ Image quality assessment for iris biometric." , In Proceedings of the 24th Annual Meeting of the Gesellscha it Klassikation, pp 445-452. Springer.

[9] Makinana, S., Malumedzha, T., Nelwamondo, F.V, (2014)" Iris Image Quality Assessment Based on Quality Parameters", Proceedings of the 6th Asian Conference on Intelligent Information and Database Systems Part I Lecture Notes in Artificial Intelligence, pp571-580. Springer,

[10] Kalka, N. D. and Zuo, J. and Schmid, N. A. and Cukic, B., (2006), "Image quality assessment for iris biometric", Defense and Security Symposium, International Society for Optics and Photonics, pp62020D-62020D

[11] Crete, F., Dolmiere, T., Ladret, P. and Nicolas, M., (2007)," The blur effect: perception and estimation with a new no-reference perceptual blur metric.”, Human Vision and Electronic Image in XII, pp6492:64920I.

[12] Sandre, S-L and Stevens, M. and Mappes, J., (2010), The effect of predator appetite, prey warning coloration and luminance on predator foraging decisions, Behaviour, vol.147, No. 9., 1121-1143, BRILL.

[13] Du, Y. and Belcher, C. and Zhou, Z. and Ives, R., (2010)," Feature correlation evaluation approach for iris feature quality measure", Signal processing, Vol. 90, No. 4, pp1176-1187, Elsevier.

[14] Nill, N. B, (2007), "IQF (Image Quality of Fingerprint) Software Application," The MITRE Corporation,

[15] Bieroza, M. and Baker, A. and Bridgeman, J., (2011),"Classification and calibration of organic matter fluorescence data with multiway analysis methods and artificial neural networks: an operational tool for improved drinking water treatment, "Environmetrics, Vol. 22, No.3, pp256-270, Wiley Online Library.

[16] Jeong, D. H. and Ziemkiewicz, C. and Ribarsky, W. and Chang, R. and Center, C. V., (2009), "Understanding Principal Component Analysis Using a Visual Analytics Tool," Charlotte Visualization Center, UNC Charlotte, 2009

[17] Suhr, D. D., (2005), "Principal component analysis vs. exploratory factor analysis," SUGI 30 Proceedings, pp 203-230.

[18] Proena, H. and Alexandre, L.A., (2005), "UBIRIS: A noisy iris image database," International Conference on Image Analysis and Processing.

[19] Chinese Academy of Sciences Institute of Automation., (2012), "CASIA Iris Database, Online:" http://http://biometrics.idealtest.org/dbDetailForUser.do?id=4. 
[20] Fauvel, M. and Benediktsson, J. A. and Chanussot, J. and Sveinsson, J. R., (2008), "Spectral and spatial classification of hyperspectral data using SVMs and morphological profiles", Geoscience and Remote Sensing IEEE Transactions on, vol. 46, No. 11, pp3804-3814.

[21] Kohavi, R., (1995), "A study of cross-validation and bootstrap for accuracy estimation and model selection,” International Joint Conferences on Artificial Intelligence, Vol 14, No. 2, pp1137-1145.

\section{AUTHORS}

Sisanda Makinana is a Biometrics Researcher at the Council for Scientific and Industrial Research (CSIR), South Africa under Information Security. She holds an MSc in Electrical Engineering from the University of Johannesburg.

Tendani Malumedzha is a Systems Engineer for Information Security at the Council for Scientific and Industrial Research (CSIR), South Africa He holds an MSc in Electrical Engineering from the University of the Witwatersrand

Fulufhelo Nelwamondo is a Competency Area Manager for Information Security at the Council for Scientific and Industrial Research (CSIR), South Africa. He holds a $\mathrm{PhD}$ in Electrical Engineering from the University of the Witwatersrand and is a visiting professor of Electrical Engineering at the University of Johannesburg.
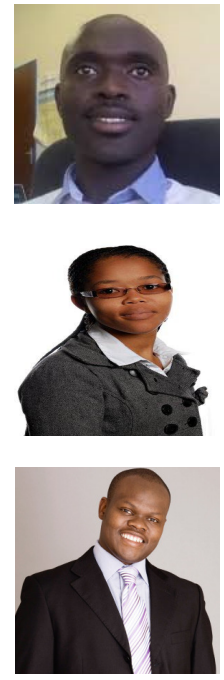